\title{
Citas de flora vascular de interés de la Sierra del Rincón (Madrid, España)
}

\author{
Jorge Baonza Díaz
}

Resumen: Baonza, J. 2012. Citas de flora vascular de interés de la Sierra del Rincón (Madrid, España). Bot. Complut. 36: 105-111.

Se citan 23 especies de flora vascular de la Sierra del Rincón (NE de la Comunidad de Madrid), de interés florístico, destacando las novedades provinciales: Adenocarpus argyrophyllus, Carex depauperata, Coeloglossum viride o Rubus canescens. Además, se han confirmado algunas especies cuya presencia en la provincia parecía dudosa o extinta como Gymnocarpium dryopteris. Destacan también las nuevas poblaciones descubiertas para especies amenazadas en la región como Ranunculus valdesii, Fraxinus excelsior, o la localización de un individuo de Sorbus intermedia.

Palabras clave: flora vascular, corología, Sierra del Rincón, Madrid.

Abstract: Baonza, J. 2012. New localities of vascular plants of interest in Sierra del Rincón (Madrid province, Spain). Bot. Complut. 36: 105-111.

New localities of 23 species of vascular plants from Sierra del Rincón (NE of Madrid province, central Spain) are signaled. There are new species to Madrid province as: Adenocarpus argyrophyllus, Carex depauperata, Coeloglossum viride or Rubus canescens. There are new populations of doubtful species in Madrid as Gymnocarpium dryopteris and regional endangered species as Ranunculus valdesii, Fraxinus excelsior, or very rare as Sorbus intermedia.

Key words: Vascular plants, corology, Sierra del Rincón, Madrid, Spain.

\section{INTRODUCCIÓN}

La Sierra del Rincón (términos municipales de Montejo de la Sierra, Prádena del Rincón, Horcajuelo de la Sierra, La Hiruela y Puebla de la Sierra) se localiza en el vértice NE de la Comunidad de Madrid, geográficamente entre las sierras de Guadarrama/Somosierra y Ayllón. Se extiende por 15.231 hectáreas entre 900 y $2047 \mathrm{~m}$ de altitud. Fue declarada por la UNESCO en 2005 como Reserva de la Biosfera, presentando importantes valores florísticos como el conocido hayedo de Montejo, único de la Comunidad de Madrid (Hernández et al. 1983). Sin embargo carece de un estudio completo de su flora vascular, contando solamente con un simple listado de la flora presente, elaborado mediante la recopilación de las citas previas sin especificar las localidades (Cuevas et al. 2005), en general ya antiguas (el último estudio importante es el mencionado catálogo del Hayedo de Montejo), aunque en 2009 hubo algunas aportaciones para la flora de la cuenca alta del Jarama (Enríquez de Salamanca 2009), compartida entre la Sierra del Rincón (La Hiruela y vertien- tes orientales de Montejo) y la provincia de Guadalajara. En julio de 2011 se pudo iniciar el estudio completo de la flora vascular de la totalidad de la reserva, con resultados espectaculares: se ha incrementado el número de especies conocidas en la reserva de unas 750 (descartadas citas duplicadas 0 erróneas) hasta cerca de 1000 (lo que está más acorde con un espacio de montaña con la superficie apuntada y que probablemente todavía se quede corto) y se han encontrado varias novedades para la Comunidad de Madrid o nuevas poblaciones de especies muy escasas en la región.

\section{MATERIALES Y MÉTODOS}

Toda el territorio está en la zona UTM 30T, por lo que no se repite en la indicación de las cuadrículas $1 \times 1 \mathrm{~km} 0$ 10 x 10 (en algunas referencias bibliográficas). El símbolo * delante de la abreviatura provincial de Madrid, M, significa novedad provincial. De la mayoría de las plantas se recolectaron pliegos depositados en el herbario del Jardín Botánico de Madrid (MA).

\footnotetext{
${ }^{1}$ Bustarviejo, 28720 Madrid.jbd@ya.com

Recibido: 29 marzo 2012. Aceptado: 9 mayo 2012.
} 


\section{RESULTADOS}

\section{Adenocarpus argyrophyllus (Rivas Goday) Caball.}

* Madrid: La Puebla de la Sierra, El Contadero, VL6044 (6043 si datum WGS84), $1725 \mathrm{~m}$, cresta cuarcítica, abundante, Baonza 11X-2011, MA. Puebla de la Sierra, VL6336, 1120 m, ladera rocosa en umbría, más de 20 pies, Baonza 25-IX-2011, MA.

La cita más cercana sería la del Valle de Iruelas (Ávila) [UK67] (Molina 1992).

\section{Angelica sylvestris L.}

MadrID: Puebla de la Sierra, Arroyo de la Puebla, VL6239, $1120 \mathrm{~m}$, juncales de la ribera, 4 ejemplares, Baonza 10-IX-2011, MA.

Se ha citado del Jarama, desde el hayedo de Montejo hasta La Hiruela (Bellot 1944, Lara et al. 1986), donde realmente parece corresponder a Angelica major (Hernández et al. 1983; datos propios) y existen citas de Somosierra (Mayor 1975; Mayor et al. 1974, dudosas al haber sido confirmada A. major (Gutiérrez 1981)), Navacerrada y el Paular (Cutanda 1861); del último lugar, en la ribera del Lozoya frente a Oteruelo del Valle y cerca del monasterio del Paular, existen citas más recientes (Fernández 1984).

\section{Carex depauperata Curtis ex With.}

* MadRID: Horcajuelo de la Sierra, VL5345, 1200 m, robledalfresneda, Baonza 21-VII-2011, MA.

Taxón que aparece disperso por la mitad norte de España, sin haber sido citado de Madrid (Luceño 2007).

\section{Chamaesyce canescens (L.) Prokh. subsp. canescens}

MadRID: Horcajuelo de la Sierra, cuneta de la carretera a Horcajo de la Sierra, VL5346, 1260 m, Baonza 7-X-2011, MA.

Taxón citado de la mitad meridional de la provincia, en el ámbito serrano solo se conocía de El Escorial, [30TVK09] (Willkomm \& Lange 1880), de las calizas de El Vellón (Gómez \& Moreno 1997, Ruiz et al. 1982) y del extremo SO provincial (Ruiz et al. 1982).

\section{Coeloglossum viride (L.) Hartm.}

* Madrid: Montejo de la Sierra, El Mosquito, VL5754, 1410 m, más de 10 ejemplares en un claro de brezal de Erica arborea (04/08/2011, sin herborizar dada la escasez de ejemplares, Fig. 1).

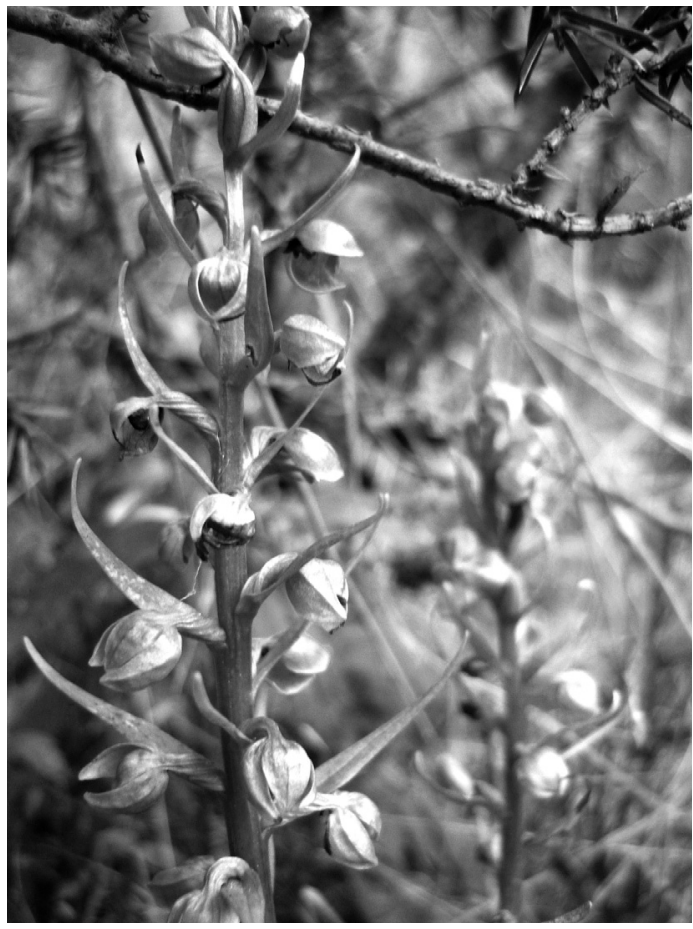

Fig. 1-Coeloglossum viride (L.) Hartm. Montejo de la Sierra, El Mosquito, 4-VIII-2011.

Señalada en la Península Ibérica de Pirineos, Cordillera Cantábrica y Sistema Ibérico, sin citar Madrid (Aedo 2005).

\section{Fraxinus excelsior L.}

MADRID: Montejo de la Sierra, Dehesa Boyal, VL5647, $1260 \mathrm{~m}$, 1 ejemplar maduro junto a la reguera procedente del hayedo, Baonza 11-X-2011, MA. Montejo de la Sierra, cabecera del río Jarama, VL5654, $1450 \mathrm{~m}$, un viejo ejemplar, 11-VIII-2011. Montejo de la Sierra, Mosquito, VL5754, 1410 m, tres ejemplares, 4-VIII-2011.

Árbol rarísismo en el hayedo de Montejo [VL5851] (Hernández \& Sáinz 1984, Hernández et al. 1983), donde se localizaron solamente dos pies (Allué et al. 1992). De zonas próximas, citada como rara en Rascafría (Fernández 1982 y 1991) y en el abedular de Canencia (Hernández \& Montero 2008), donde podría ser naturalizado (datos propios). A principios del siglo XX plantado en el monte la Jurisdicción de San Lorenzo de El Escorial, donde todavía quedan algunos pies en barrancos (Allué et al. 1992). Más frecuente en la vertiente segoviana y guadalajareña de la Sierra de Ayllón (Allué et al. 1992; Hernández \& Sáinz 1984; Lara et al. 1996). 


\section{Glechoma hederacea L.}

Madrid: Montejo de la Sierra, Río de la Madre, VL5545, 1100 m, fresneda, Baonza, 9-VIII-2011, MA. Puebla de la Sierra, Arroyo de la Puebla, VL6239, juncales de la ribera, muy escaso, Baonza, 10IX-2011, MA.

En Madrid con dos citas del s. XIX de Lozoya y Guadarrama (Gamarra 1992; Morales \& Gamarra 1990; Ruiz et al. 1982), un pliego sin fecha de El Paular (Morales 1998) y citas más recientes en Montejo, VL5853 (Ruiz et al. 1982) y la vertiente segoviana de la Sierra de Guadarrama (Romero \& Rico 1989).

\section{Gymnocarpium dryopteris (L.) Newman}

Madrid: Montejo de la Sierra, El Mosquito, VL5754, $1430 \mathrm{~m}$, abedular de B. pubescens, Baonza, 4-VIII-2011, MA.

Localidad cercana a la segoviana de Cerezo de Arriba, VL5860 (Romero \& Rico 1989), provincia de donde se citó del Puerto de Navacerrada (Leresch \& Levier 1880, en Rivas et al. 1981). En Madrid habría sido citado de Guadarrama [¿municipio, sierra?] (Lázaro, en Rivas 1964, aunque ni su «Compendio de Flora Española», $3^{\text {a }}$ ed., tomo I, 1920, donde lo señala de las montañas del N y NE, ni sus «Contribuciones a la flora de la Península Ibérica I y II», 1893 y 1900 en los Anales de la Sociedad Española de Historia Natural, recogen tal referencia), y se consideraba dudosa en esta provincia (Salvo \& Otermin 1986).

\section{Lithospermum officinale $\mathrm{L}$.}

MADRID: Prádena del Rincón, VL5641, 1170 m, sauceda mixta (S. fragilis, S. atrocinerea, Populus tremula, etc.), Baonza, 7-IX-2011, MA.

En Madrid citada de El Atazar (Fuente 1985) y la Casa de Campo (Cutanda 1861, en Ruiz et al. 1982).

\section{Lobelia urens $\mathrm{L}$.}

Madrid: Puebla de la Sierra, Los Regajos del Prado, VL6038, 1270 m, arroyuelo, 8-IX-2011. Puebla de la Sierra, VL6137, 1090 m, juncal en cuneta de la carretera, Baonza, 6-IX-2011, MA. Puebla de la Sierra, VL6236, 1140 m, juncal, 14-VII-2011. Puebla de la Sierra, VL6337, $1090 \mathrm{~m}$, pequeño juncal cortado por pista forestal, 25-IX2011.

Citada en Madrid antiguamente de San Martín de Valdeiglesias (Quer, en Ruiz et al. 1982) y escasa de Somo- sierra, [30TVL55] (Rivas 1964); también de zonas cercanas de Guadalajara (Fuente 1985).

\section{Paeonia officinalis L. subsp. microcarpa}

(Boiss. \& Reut.) Nyman

MADRID: Horcajuelo de la Sierra, Dehesa de Horcajuelo, VL5246, 1330 m, robledal de Q. pyrenaica de umbría, 21-VII-2011. Prádena del Rincón, Dehesa de Ana Gutiérrez, VL5442, 1080 m, robledal Q. pyrenaica con Fraxinus angustifolia, convive con P. broteroi, Baonza, 9VII-2011, MA.

Citada recientemente de Montejo de la Sierra, VL5747 (Enríquez de Salamanca 2009); en Madrid se conoce además de Rozas de Puerto Real, UK7066 (Morales \& Fernández 1990), Guadalix de la Sierra (Ruiz et al. 1982) y con citas del siglo XIX del Puerto de Guadarrama, [VL0307] y Carabaña [VK85] (Lázaro 1893).

\section{Phyteuma spicatum L.}

MADRID: Montejo de la Sierra, alto Jarama, VL5654, 1420 m, abedular de B. pubescens, escasa, Baonza, 11-VIII-2011, MA.

Citada antiguamente en Madrid de Somosierra y El Paular (Quer, Cutanda, Isern, en Ruiz et al. 1982) recientemente encontrado en La Hiruela, riberas del Jarama, 1160 m, VL6348, muy rara (Enríquez de Salamanca 2009). Conocido de la vertiente segoviana de la Sierra de Guadarrama, Navafría (García 1995).

\section{Potamogeton trichoides Cham. \& Schlecht.}

Madrid: Puebla de la Sierra, VL6241, $1220 \mathrm{~m}$, balsa artificial, abundante, Baonza, 13-X-2011, MA.

En Madrid conocido de charcas y ríos de la cuenca del río Guadarrama y del Manzanares, VK19 y VK39 (Arnáiz \& Molina 1985).

\section{Quercus robur L.}

MADRID: La Hiruela, ladera rocosa de umbría junto al Jarama, VL6448, 1130 m, 2 ejemplares puros, Baonza, 19-VII-2011, MA y 3 Q. $\times$ andegavensis Hy (Q. pyrenaica x Q. robur), Baonza, 19-VII-2011, MA.

Confirma una antigua cita de la dehesa boyal de La Hiruela (Madariaga 1909, en Allué et al. 1992), quien también lo citó del Hayedo de Montejo, donde ha sido señalado más recientemente, VL5853 (Ruiz et al. 1982). Además se conoce de la Dehesa de Somosierra, VL5153 
(Romero \& Rico 1989); de Puebla de la Sierra, monte número 109 del C.U.P. [Dehesa Boyal, VL63], dos ejemplares bastante típicos y otros con influencia de $Q$. pyrenaica, e incluso de Q. petraea (Allué et al. 1992). Como cultivado y a veces subespontáneo en El Escorial, Villaviciosa de Odón, El Pardo, Casa de Campo, Retiro y Aranjuez (Willkomm \& Lange 1861, Ruiz et al. 1982; López 1984). En algunos de estos lugares, los Reales Sitios, fueron plantados robles procedentes de Flandes en tiempos de Felipe II (Puerto 2003: 369). Citado en la vertiente guadalajareña de la Sierra de Ayllón, en Cantalojas (Castel 1883; Ceballos 1966, en Allué et al. 1992).

\section{Ranunculus valdesii Grau}

MADRID: Montejo de la Sierra, alto Jarama, VL5654 (5754 si datum Europeo 1950), $1420 \mathrm{~m}$, abedular de B. pubescens, escaso, Baonza 11-VIII-2011, MA

Especie descrita de la vertiente madrileña de la Sierra de Guadarrama, en melojares de la subida al Puerto del Reventón [VL22] (Grau 1984, Cook et al. 1986); citado cerca en la vertiente segoviana de la misma sierra (García 1995) y de la Sierra de Ayllón (Hernández \& Sainz 1984, sub. R. auricomus; Romero \& Rico 1989).

\section{Rosa arvensis Huds.}

MADRID: Horcajuelo de la Sierra, Dehesa de Horcajuelo, VL5246, $1330 \mathrm{~m}$, robledal de Q. pyrenaica de umbría, 21-VII-2011. Horcajuelo de la Sierra, VL5346 (5245 si datum WGS84), $1260 \mathrm{~m}$, robledal de $Q$. pyrenaica, 7-X-2011). Montejo de la Sierra, Dehesa Boyal, VL5647, 1230-1260 m, robledal de Q. pyrenaica, abundante, 30-VII-2011. Prádena del Rincón, VL5744, 1180 m, robledal de Q. pyrenaica de umbría, 12-VII-2011. La Hiruela, Dehesa de la Hiruela, VL6347, 1270 m, robledal de Q. pyrenaica, var arvensis, abundante, Baonza 17-IX-2011, MA, y var. atrata (H. Crist) Boulenger, Baonza, 17-IX-2011, MA, rara.

Citada en Madrid del hayedo de Montejo [VL5851] (Hernández et al. 1983) y del valle de Bustarviejo (Baonza 2004), donde está más ampliamente distribuida de lo señalado en la cita.

\section{Rosa pimpinellifolia $\mathrm{L}$.}

* Madrid: Horcajuelo de la Sierra, Dehesa de Horcajuelo, VL5246, $1330 \mathrm{~m}$, robledal de $Q$. pyrenaica de umbría, 1 mata, Baonza, 21-VII-2011, MA.

Las citas más cercanas son de Majalrayo (Guadalajara), VL75 (Mayor 1975: 330), Consuegra (Segovia) VL36
(Rivas 1969, sub. R. spinosissima) y de la Sierra de Pradales (Segovia), VL3883 (Romero \& Rico 1989).

\section{Rubus canescens DC.}

* MadRID: Horcajuelo, VL5345, $1230 \mathrm{~m}$, fresneda aclarada, convive con R. ulmifolius y R. lainzii, Baonza, 21-VII-2011, MA. Montejo de la Sierra, VL5545, $1130 \mathrm{~m}$, setos de los prados con R. ulmifolius, 9-VIII-2011. Montejo de la Sierra, Dehesa Boyal, VL5647, $1230 \mathrm{~m}$, fresneda adehesada, convive con R. castellarnaui, R. ulmifolius y $R$. lainzii, con este último parece hibridar, 30-VII-2011.

Las citas más cercanas son del Alto Tajo, Guadalajara (Monasterio 1990) aunque parece que fue citado de la Sierra de Guadarrama (Graells, en Ruiz et al. 1982).

\section{Sorbus intermedia (Ehrh.) Pers.}

MADRID: Montejo de la Sierra, Ribera del Jarama, VL5753, 1340 m, 1 pie junto a varios $S$. aria, cerca abunda $S$. aucuparia, Baonza 4VIII-2011, MA.

En la provincia se conoce sólo de La Pedriza (Cantero 2008). Dado que en Montejo solo hay un individuo junto a los parentales de la especie (de origen híbrido), tal vez sería más correcto hablar de $S$. aria x $S$. aucuparia mientras no se pueda confirmar que el individuo es fértil, ya que no se observaron frutos.

\section{Solanum physalifolium Rusby}

MadRID: Montejo de la Sierra, VL5545 (5445 si datum WGS84), $1160 \mathrm{~m}$, mala hierba de las huertas, Baonza 9-VIII-2011, MA. Puebla de la Sierra, VL6240, 1130 m, mala hierba de las huertas, Baonza, 2VIII-2011, MA.

Especie alóctona citada en Madrid en Flora Iberica (Sobrino \& Sanz 2012), no se ha encontrado otra cita de la provincia.

\section{Ulmus glabra Huds.}

MADRID: Puebla de la Sierra, VL6336, 1120 m, ladera de umbría con humedad edáfica, 1 ejemplar, Baonza, 25-IX-2011, MA. Hayedo de Montejo, VL5753, 1430 m, 2 pies en acebeda, 11-VII-2011. Montejo de la Sierra, El Mosquito, VL5654, 1570-1460 m, 3 ejemplares dispersos, 11-VIII-2011.

Citado como muy raro en la zona superior del hayedo de Montejo, VL5752 (Rivas 1964, Mayor 1975, Hernán- 
dez \& Sáinz 1984, Hernández et al. 1983, Rossignoli \& Génova 2003, la cuadrícula VL5554 recogida por Ruiz et al. 1982 atribuída a Rivas Martínez es errónea), la garganta del arroyo de Santa Ana en Oteruelo del Valle (Rascafría) y Alameda del Valle (Baonza 1996, Rossignoli \& Génova 2003, Enciso 2008), Valle de la Fuenfría (Ruiz et al. 1982, Rossignoli \& Génova 2003) y Rozas de Puerto Real, UK7263 (Gómez 1989, Rossignoli \& Génova 2003). Fuera de la provincia de Madrid, en la Sierra de Ayllón (Mayor 1965, Hernández \& Sáinz 1984, Allué et al. 1992, Lara et al. 1996, Rossignoli \& Génova 2003) y en la vertiente segoviana de la Sierra de Guadarrama (García 1995; Rossignoli \& Génova 2003).

\section{Valeriana officinalis L. subsp. officinalis}

MADRID: Montejo de la Sierra, Río Jarama, VL5852, $1300 \mathrm{~m}$, abedular de B. pubescens, escaso, 4-VIII-2011. Montejo de la Sierra, El Mosquito, VL5754, $1410 \mathrm{~m}$, brezal de $E$. arborea con arbolado, Baonza, 4-VIII-2011, MA.

Citada antiguamente de Miraflores, Chozas [Soto del Real], El Paular y Valle del Lozoya (Quer, Colmeiro, en Willkomm \& Lange 1880 y por Ruiz et al. 1982), de los dos últimos lugares ha sido confirmada más recientemente su presencia (Fernández 1991, sub. V. procurrens); se conoce además de Somosierra (Bellot 1944, sub. V. sambucifolia; Peinado et al. 1987).

\section{Viola montcaunica Pau}

MAdRID: Horcajuelo de la Sierra, La Garita, VL5452, $1650 \mathrm{~m}$, juncal en ladera, rara, Baonza, 28-VII-2011, MA.

En Madrid únicamente citada de zonas cercanas como la Dehesa de Somosierra, [VL5152] (Cutanda 1861),
Cerro de Cebollera, Somosierra, [VL5457] (Pau 1915) y en la zona superior del hayedo de Montejo [VL5751] (Hernández et al. 1983).

\section{DISCUSIÓN}

La Sierra del Rincón se presenta como la principal zona de flora eurosiberiana de la Comunidad de Madrid, de la que el más destacado ejemplo sería el conocidísimo Hayedo de Montejo. Otro paraje notable sería el Mosquito: ladera de umbría sobre el nacimiento del Jarama, dominado por un bosque mixto de Sorbus aucuparia, Betula pubescens (enclaves higrófilos), Ilex aquifolium, Taxus baccata, Quercus pyrenaica, Q. petraea, Fraxinus excelsior, Ulmus glabra, Prunus avium y Sambucus nigra y con herbáceas de gran interés regional como $R a$ nunculus valdessii, Gymnocarpium dryopteris, Trollius europaeus, Streptopus amplexifolius, Phyteuma spicatum, Valeriana officinalis, Paris quadrifolia, etc. En tal paraje servales, acebos y tejos son capaces de colonizar vigorosamente las plantaciones de Pinus sylvestris en terrazas situadas por encima. También se puede destacar la sorprendente presencia, principalmente en el término de Puebla de la Sierra, de flora que para Madrid denota influencia atlántica como Osmunda regalis (Moreno \& Fernández 1982), Erica scoparia (Allúe \& García 1990), Lobelia urens y a las que se puede añadir Adenocarpus argyrophyllus.

\section{AGRADECIMIENTOS}

A Ángel Quirós, por acompañarme en algunas visitas por la zona, y a la D. G. de Medio Ambiente, Área de Educación Ambiental de la Consejería de Medio Ambiente, Vivienda y Ordenación del Territorio de la Comunidad de Madrid, que ha financiado el inicio del proyecto.

\section{BIBLIOGRAFIA}

Aedo, C. 2005. Coeloglossum Hartm. En C. Aedo \& A. Herrero (Eds.) Flora Iberica, 21: 92-94. Real Jardín Botánico, Madrid.

Allué, M. \& García-LóPez, J. M., 1990. Sorbus torminalis (L.) Crantz, novedad en la provincia de Madrid. Anales Jard. Bot. Madrid 48 (1): 85-86. Madrid.

Allué, M.; García-LóPez, J. M.; Ruiz Del Castillo, J.; Ruiz De La Torre, J. \& Martínez-Labarga, J. M. 1992. Notas sobre flora y vegetación en el sector oriental del Sistema Central. Ecología 6: 51-65.
Arnáiz, C. \& Molina, J. A. 1985. Vegetación acuática y helofítica de la cuenca alta del río Guadarrama (Madrid, España). Lazaroa 8: 221-240.

BaONZA, J. 1996. Viscum album subsp. album en Madrid. Ecología 10: 317-319.

BaONZA, J. 2004. Algunas plantas de interés del Sistema Central: Nuevas citas guadarrámicas y revisión corológica. Ecología 18: 215-224.

BeLLOT, F. 1944. Estudio sobre la vegetación y la flora de la comarca de Somosierra. Anales R. Acad. Farmacia 10: 109-130. 
Cantero Desmartines, F. J. 2008. Sorbus intermedia (Ehrh.) Pers. (Sorbus mougeotii Soy.-Will. et Godr.), novedad para el Sistema Central. Foresta 41: 70-79.

Cook C. D. K.; Grau, J. \& López, G. 1986. Ranunculus L. En Castroviejo et al. (Eds.) Flora Iberica, 1: 279-371. Real Jardín Botánico, Madrid.

Cuevas, J. A.; de las Heras, P.; López, N. \& Roldán, M. J. 2005. Caracterización paisajística y ecológica de la Sierra del Rincón (Madrid). Centro de Investigaciones Ambientales de la Comunidad de Madrid.

Cutanda, V. 1861. Flora compendiada de Madrid y su provincia. Imprenta Nacional, Madrid.

Enciso Encinas, E. 2008. Fuentes semilleras de la Comunidad de Madrid, tomo II, Consejería Medio Ambiente y Ordenación del Territorio, Madrid.

Enríquez de Salamanca SÁnchez-CÁmara, A. 2009. Adiciones y correcciones a la flora de la cuenca alta del río Jarama (Guadalajara y Madrid). Flora Montiberica 42: 55-64.

FERnÁNDEZ GonZÁLEZ, F. 1982. Notas florísticas sobre el Valle del Paular II. Lazaroa 4: 375-378.

FernÁndez GonZÁLEZ, F. 1984. Notas florísticas sobre el Valle del Paular III. Lazaroa 6: 271-274.

FernÁndez GonzÁLez, F. 1991. La vegetación del valle del Paular (Sierra de Guadarrama, Madrid), I. Lazaroa 12: 153272. Madrid

Fuente, V. DE LA 1985. Vegetación orófila del occidente de la provincia de Guadalajara (España). Lazaroa 8: 123-219.

Gamarra, R. 1992. Adiciones. Mapa 413. Fontqueria 33: 191.

García AdÁ, R. 1995. Estudio de la flora y vegetación de las cuencas alta y media de los ríos Eresma, Pirón y Cega (Segovia). Tesis de licenciatura, Fac. Biología, Univ. Complutense, Madrid.

Gómez Manzaneque, F. 1989. Algunos táxones interesantes del suroeste madrileño. Stud. Bot. 7: 257-261.

Gómez Manzaneque, F. \& Moreno SÁiz, J. C. 1997. Catálogo de la flora vascular de las calizas cretácicas de Soto del RealSan Agustín de Guadalix (Madrid). Ecología 11: 207-234.

GraU, J. 1984. Vorläufige Übersicht iberischen Vertreten von Ranunculus sect. auricomus. Mitt. Bot. Staatssamml. München 20: 11-28.

Gutiérrez Bustillo, A. M.1981. Revisión del género Angelica L. (Umbelliferae) en la Península Ibérica. Lazaroa 3: 137-162.

Hernández, J. E.; Costa, M.; SÁinz, H. \& Clemente, M. 1983. Catálogo florístico del Hayedo de Montejo de la Sierra (Provincia de Madrid). Lagascalia 11(1): 3-65.

Hernández, A. \& Montero, F. 2008. Monte de utilidad pública perimetro de Canencia. Cien años de gestión forestal. Dir. Gral. de Medio Ambiente, Consejería Medio Ambiente, Vivienda y Ordenación Territorio. Madrid.

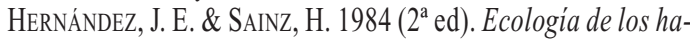
yedos meridionales ibéricos: el macizo de Ayllón. IEAPA. Madrid.

Lara, F.; Garilleti, R. \& Ramírez, P. 1996. Estudio de la vegetación de los ríos carpetanos de la cuenca del Jarama. CEDEX, Madrid.
LÁzaro IbizA, B. 1893. Contribuciones a la flora de la Península Ibérica. Notas críticas acerca de la flora española (Primera serie). Anales Soc. Esp. Hist. Nat. 22(1): $19-46$.

López Lillo, A. 1984. Árboles de Madrid. Comunidad de Madrid.

LuCEÑo, M. 2007. Carex L. En Castroviejo, S. et al. (Eds.): Flora Iberica, 18: 109-250. Real Jardín Botánico, Madrid.

MAYOR, M. 1965. Estudio de la flora y vegetación de las sierras de Pela, Ayllón y Somosierra. Tesis doctoral Universidad Complutense, Madrid.

Mayor, M. 1975. Datos florísticos sobre la Cordillera Central (Somosierra, Ayllón y Pela). Anales Inst. Bot. Cavanilles 32(2): 323-347.

Mayor, M.; Díaz, T. E.; NAVArro, F.; Martínez, G. \& Andrés, J. 1974. Los pastizales del Sistema Central. Nota I: Somosierra, Ayllón y Pela. Revista Facultad Ciencias Universidad Oviedo 16: 283-322.

Molina Moreno, J. R. 1992. Flora y vegetación del valle de Iruelas (Ávila). Cuadernos Abulenses 18: 11-149.

Monasterio-Huelin, E. 1990. Notas sobre Rubus ibéricos. Anales Jard. Bot. Madrid 47(2): 521-523.

Morales Abad, M.J. \& Fernández Casas, J. 1990. Asientos para un atlas corológico de la flora occidental. Mapa 402. Fontqueria 28: 115-118.

Morales, R. 1998. Las labiadas de la Comunidad de Madrid. Bot. Complut. 22: 9-62.

Morales Abad, M.J. \& Fernández Casas, J. 1990. Asientos para un atlas corológico de la flora occidental. Mapa 402. Fontqueria 28: 115-118.

Morales Valverde, R. \& Gamarra, R. 1990. Asientos para un atlas corológico de la flora occidental. Mapa 413. Fontqueria 28: $135-136$.

Moreno, J. M. \& F. Fernández. 1982. Osmunda regalis L. en la Sierra de Madrid (España). Lazaroa 4: 391-392.

Pau, C. 1915. Notas sueltas sobre la flora matritense. Bol. Soc. Aragonesa Ci. Nat. 14: 204-211.

Peinado, M.; Moreno, G. \& Bartolomé, C. 1987. Datos florísticos y ecológicos sobre los abedules del Sistema Central. Publicaciones de la Universidad de La Laguna. Serie Informes 22: 207-216.

Puerto, J. 2003. La leyenda verde. Naturaleza, sanidad y ciencia en la corte de Felipe II (1527-1598). Junta de Castilla y León. Salamanca.

Rivas Martínez, S. 1964. Estudio de la vegetación y flora de las Sierras de Guadarrama y Gredos. Anales Inst. Bot. Cavanilles 21(1): 5-325.

Rivas MartíneZ, S. 1969. Vegetatio Hispaniae. Notula I. Publ. Inst. Biol. Apl. 46: 5-34.

Rivas Martínez, S. 1979. Brezales y jarales de Europa occidental (revisión fitosociológica de las clases Calluno-Ulicetea y Cisto-Lavanduletea). Lazaroa 1: 1-127.

Rivas Martínez, S.; Costa, M.; Izco, J. \& SÁenz, C. 1981. Flora Matritensis, I (Pteridophyta). Lazaroa 3: 25-61

Romero, T. \& Rico, E. 1989. Flora de la cuenca del río Duratón. Ruizia 8: 7-438. 
Rossignoli, A. \& GÉNOVA, M. 2003. Corología y hábitat de Ulmus glabra Huds. en la Península Ibérica. Ecología 17: 99-121.

Ruiz, J. (Dir.); Abajo A.; CARmona E.; Escribano R.; Ortega C.; Rodríguez, A. \& Ruiz, J. 1982. Aproximación al catálogo de plantas vasculares de la provincia de Madrid. Consejería de Agricultura y Ganadería, Madrid.

Salvo, A. \& Otermin, P. 1986. Gymnocarpium Newman. En
Castroviejo et al. (Eds.) Flora Iberica 1: 121-123. Real Jardín Botánico, Madrid.

Sobrino Vesperinas, E. \& SAnz Elorza, M. 2012. Solanum L. En Talavera et al. (eds.) Flora Iberica 11: 166-195. Real Jardín Botánico, Madrid.

Willkomm, M. \& Lange, J. 1861-1880. Prodromus florae Hispanicae, 1-3. Sttutgart. 